\title{
Application of an Advanced Image-Based Virtual Monoenergetic Reconstruction of Dual Source Dual-Energy CT Data at Low keV Increases Image Quality for Esophageal Cancer Patients Imaging
}

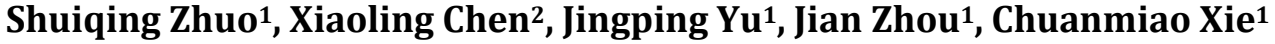 \\ ${ }^{1}$ Department of Imaging Sciences of Sun Yat-sen University Cancer Center, State Key Laboratory of Oncology in South China, \\ Collaborative Innovation Center for Cancer Medicine, Guangzhou, China \\ ${ }^{2}$ The First Affiliated Hospital of Sun Yat-sen University, Guangzhou, China \\ Email: zhuoshq@sysucc.org.cn
}

How to cite this paper: Zhuo, S.Q., Chen, X.L., Yu, J.P., Zhou, J. and Xie, C.M. (2018) Application of an Advanced Image-Based Virtual Monoenergetic Reconstruction of Dual Source Dual-Energy CT Data at Low $\mathrm{keV}$ Increases Image Quality for Esophageal Cancer Patients Imaging. Open Journal of Medical Imaging, 8, 81-88.

https://doi.org/10.4236/ojmi.2018.83010

Received: August 23, 2018

Accepted: September 23, 2018

Published: September 26, 2018

Copyright $\odot 2018$ by authors and Scientific Research Publishing Inc. This work is licensed under the Creative Commons Attribution International License (CC BY 4.0).

http://creativecommons.org/licenses/by/4.0/

\section{c) (i) Open Access}

\begin{abstract}
Purpose: To explore the significance of dual-source computed tomography (DECT) virtual monoenergetic reconstructions technology in improving the imaging quality of esophageal cancer patients. Materials and methods: 68 patients with clinically suspected esophageal cancer (all confirmed by pathology) were collected. Routine plain scan was performed with SIEMENS Force dual-energy CT and then dual-phase scans were performed. The venous phase images were respectively subjected to traditional virtual monoenergetic reconstructions (Mono_E) and new generation virtual monoenergetic reconstructions (Mono+). Mono_E $55 \mathrm{keV}$ and Mono+ $55 \mathrm{keV}$ virtual single-energy images were obtained respectively. The signal-to-noise ratio (SNR) of normal esophageal tissue and esophageal cancer lesions, noise and contrast noise ratio (CNR tumor) of normal esophageal tissue and esophageal cancer lesions were compared among $100 \mathrm{kV}$ images, Mono_E images and Mono+ images. At the same time, two imaging physicians read the films and scored the images of each group by using a 5-point scoring method. Results: Mono+ $55 \mathrm{keV}$ images, SNR, SNRtumor, noise and CNRtumor were statistically different from those of $100 \mathrm{kV}$ images and Mono_E images $(\mathrm{P}<0.05)$. And Mono+ $55 \mathrm{keV}$ images also had the highest subjective score, with statistical significance $(\mathrm{P}<0.05)$. The results showed that Mono+ $55 \mathrm{keV}$ images had the best quality. Conclusion: The new generation of virtual monoenergetic reconstructions post-processing (Mono+) could reduce image noise and improve the contrast between esophageal cancer lesions and normal esopha-
\end{abstract}


geal tissues, which was of great significance to improve the imaging quality of esophageal cancer patients and improve the early detection rate of esophageal cancer.

\section{Keywords}

Dual-Source, Dual-Energy, Virtual Monoenergetic Reconstructions, Computed Tomography, Esophageal Cancer

\section{Introduction}

Esophageal cancer is a common fatal malignant tumor. The Chinese incidence rate of esophageal cancer ranks the fourth in the world [1]. Esophageal cancer has hidden onset and late appearing clinical symptoms. As a result, most of the clinical patients have reached the middle and late stage, and their 5-year survival rates after treatment are very low [2]. The early diagnosis and treatment of esophageal cancer are of great significance to the surgical resection rate of esophageal cancer lesions and the improvement of survival rate of patients. The purpose of this study is to explore the advantages of dual-source dual-energy CT new-generation virtual single-energy post-processing technology (Mono+) compared with $100 \mathrm{kV}$ mixed-energy images and traditional virtual single-energy post-processing technology (Mono_E) and its significance in improving the imaging quality of esophageal cancer patients.

\section{Materials and Methods}

\subsection{General Data}

68 patients with esophageal cancer from July 2016 to June 2018 were retrospectively collected, including 58 males and 10 females, aged 46 to 78 years old. Their average age was $59.15 \pm 7.83$ years old. The median age was 59.5 years old, and the average weight was $58.02 \pm 6.89 \mathrm{~kg}$. All of them were confirmed by pathological results, including 13 cases of poorly differentiated squamous cell carcinoma, 13 cases of highly differentiated squamous cell carcinoma, 41 cases of moderately differentiated squamous cell carcinoma and 1 case of adenocarcinoma. There was no statistical difference in patients' weights $(P<0.05)$. Case inclusion criteria: 1) All the patients were initially diagnosed;2) All patients with pancreatic cancer confirmed by pathological results; 3) The patients were conscious and able to cooperate with the examination. Exclusion criteria: 1) The patients have hepatic and renal insufficiency; 2) The patients have contraindication of iodinated contrast agent; 3) Have serious cardiac dysfunction and other diseases.

\subsection{Inspection Methods}

All study examinations were performed on the same third-generation dual-source 
CT scanner (SOMATOM Force, Siemens Healthcare, Forchheim, Germany); the U.S. MEDRAD Stellant CT special high pressure syringe was used. The contrast agent was $350 \mathrm{mg} / \mathrm{ml}$ nonionic iodine-containing contrast agent. The dosage was $1.5 \mathrm{ml} / \mathrm{kg}$ body weight and the bolus injection rate was $3 \mathrm{ml} / \mathrm{s} .20 \mathrm{ml}$ saline was injected at the same speed to test the patency of blood vessels before bolus injection of contrast agent, and $20 \mathrm{ml}$ saline was injected after bolus injection of contrast agent. All cases underwent conventional plain scan, arterial and venous dual energy scan. In the arterial phase, the contrast bolus intelligent tracking technique was used. The tracking point was set at the central part of the descending aorta at the bifurcation level of the trachea. The ROI area was about $1 \mathrm{~mm}^{2}$ and the trigger threshold was $100 \mathrm{HU}$. The arterial phase scan was started 7 seconds after the enhancement of the region of interest reached the threshold. The scanning range is from the first rib to the bottom of the lung, and the scanning time is about 3 seconds. The portal phase scan was triggered 28 seconds after the arterial phase was completed. During venous phase, double energy scanning was used to start the A and B bulb tubes. Scanning parameters: CARE Dose4D tube voltage and tube current intelligent modulation technology was used. The reference tube voltage/tube current of the A and B bulb tubes were: $100 \mathrm{kV} / \mathrm{ref} 250 \mathrm{mAs}$ and $\mathrm{Sn} 150 \mathrm{kV} / \mathrm{ref} 125 \mathrm{mAs}$. The bulb tube rotation time, 0.5 second, pitch 0.9; collimation: $2 \times 192 \times 0.6 \mathrm{~mm}$; thickness slice: $1 \mathrm{~mm}$.

\subsection{Image Reconstructions}

The three groups of images $\mathrm{A}, \mathrm{B}$, and $\mathrm{M}$ obtained by intravenous dual energy scanning respectively represent $100 \mathrm{kV}$ images, $\mathrm{Sn} 150 \mathrm{kV}$ images, and linear fusion images of the first two. Workstations that transmit images A and B are post-processed by using the "dual energy" function module in Syngo.via software. Two groups of images were selected for post-processing by traditional Mono_E and new generation of single energy Mono+ respectively to obtain two groups of virtual single-energy images including Mono_E $55 \mathrm{keV}$ and Mono+ $55 \mathrm{keV}$.

\subsection{Image Analysis and Measurement}

Subjective evaluation: Syngo.via software was used for film reading in Siemens workstation. 2 senior imaging diagnostic physicians used double blind method respectively gave subjective evaluation for the above 3 groups of images. In case of disagreement, joint review and consultation would be performed to reach agreement. The window width was $400 \mathrm{HU}$ and the window level was $50 \mathrm{HU}$. The structure of the evaluation included normal esophageal and tumor tissue display and overall image. Imaging quality evaluation criteria included: image noise, resolution, contrast, sharpness, artifacts, and the display of the edge of the lesion contour and the lesions. 5-point evaluation method was adopted: 1 point: the image quality is very poor and cannot be used for evaluation at all (the image noise is very high; the artifact is extremely significant; the spatial or contrast res- 
olution is completely distorted, and the edge definition is very poor); 2 points: the image quality is poor, and can basically be used for evaluation (image noise is high; the artifact is obvious; spatial or contrast resolution is distorted, and edge definition is poor); 3 points: the image quality is general and can be tolerated, but is affected by appropriate amount of noise, artifacts and image distortion; 4 points: the image quality is good. The noise, artifact and image distortion does not affect the clinical diagnosis; 5 points: the image quality is very good. There is low noise, good contrast, no artifact and no image distortion, which completely meets the clinical diagnostic requirements.

Objective evaluation: Syngo.via software "MM Basic Comparison" was used to read the function module. At the same time, the above three groups of simulated mono-functional images and $100 \mathrm{Kv}$ images were opened. The background noise of the images and the CT values and standard deviation of normal esophageal tissue and tumor tissue were measured. Measurement position: three groups of images were selected at the same level. ROI was placed in the air in front of the images, normal esophageal tissue and tumor parenchyma respectively. The ROI was about 0.3 to $1 \mathrm{~mm}^{3}$. Each part was measured 3 times and the average value was taken 3 times. The signal-to-noise ratio (SNR) of normal esophagus and tumor and the contrast noise ratio $\mathrm{CNR}_{\text {tumor }}$ of tumor were calculated. The calculation formula was: $\mathrm{SNR}=\mathrm{ROI} / \mathrm{SD}, \mathrm{CNR}_{\text {tumor }}=\left(\mathrm{ROI}_{\text {eso }}-\mathrm{ROI}_{\text {tumor }}\right) / \mathrm{Sd}_{\text {air }}$.

\subsection{Statistical Analysis}

The measurement data of each group was expressed as mean standard deviation. SPSS 20 software was adopted for analysis. Comparison of the counting data was performed by $X^{2}$ test. Comparison of the measurement data was performed by $\mathrm{t}$ test, with $\mathrm{P}<0.05$ indicating statistically significant difference. Kappa consistency analysis was used for the image scoring of the two radiologists. Kappa $\geq$ 0.75 indicated good consistency; $0.40 \leq$ Kappa $<0.75$ indicated relatively good consistency; Kappa $<0.40$ indicated poor consistency.

\section{Results}

The signal-to-noise ratio (SNR) of normal esophagus and tumor, contrast noise ratio (CNR tumor) of tumor, noise air measurement data and statistical results of the three groups of images are shown in Table 1 . And subjective scoring results of image quality were shown in Table 2.

\section{Results Analysis}

The results of objective evaluation in Table 1 showed that there were significant differences in normal esophageal signal-to-noise ratio (SNRESO), tumor tissue signal-to-noise ratio (SNRtumor), background noise (NOISEair) and tumor tissue contrast noise ratio (CNRtumor) of 3 Group between either two groups of the three groups $(\mathrm{P}<0.05)$. Among them Mono_E $55 \mathrm{keV}$ image had the lowest SNReso, SNRtumor and CNRtumor, and the highest NOISEair. Mono+ $55 \mathrm{keV}$ 
Table 1. 3 Statistical results of three groups of image measurement data $(x \pm s)$.

\begin{tabular}{ccccc}
\hline & $100 \mathrm{kV}$ & Mono_E $55 \mathrm{keV}$ & Mono+55 keV & P value \\
\hline SNReso & $3.93 \pm 1.40$ & $2.37 \pm 0.82$ & $4.60 \pm 1.49$ & $\mathrm{P}<0.05$ \\
SNRtumor & $3.62 \pm 0.85$ & $2.37 \pm 0.71$ & $4.15 \pm 0.99$ & $\mathrm{P}<0.05$ \\
NOISEair & $7.30 \pm 1.51$ & $16.06 \pm 2.85$ & $6.09 \pm 1.03$ & $\mathrm{P}<0.05$ \\
CNRtumor & $2.47 \pm 2.19$ & $1.78 \pm 1.70$ & $4.27 \pm 3.83$ & $\mathrm{P}<0.05$ \\
\hline
\end{tabular}

Table 2. 3 Subjective scoring results of imaging quality of three groups $(\bar{x} \pm s)$.

\begin{tabular}{cccc}
\hline & $100 \mathrm{kV}$ & Mono_E $55 \mathrm{keV}$ & Mono+55 keV \\
\hline Normal esophageal display & $4.02 \pm 0.52$ & $2.75 \pm 0.55$ & $4.47 \pm 0.54$ \\
Tumor contour display & $3.89 \pm 0.22$ & $2.68 \pm 0.52$ & $4.57 \pm 0.52$ \\
Imaging quality & $3.65 \pm 0.33$ & $2.70 \pm 0.53$ & $4.28 \pm 0.41$ \\
\hline
\end{tabular}

images had the highest SNReso, SNRtumor and CNRtumor, and the lowest NOISEair. The results of subjective evaluation in Table 2 showed that the subjective score of Mono_E $55 \mathrm{keV}$ image was the lowest in normal esophagus, tumor tissue and image overall quality, with significant differences compared with other 2 groups $(\mathrm{P}<0.05)$; while the subjective score of Mono+ $55 \mathrm{keV}$ images was the highest, with significant differences compared with other 2 groups $(\mathrm{P}<$ $0.05)$.

\section{Discussion}

With the wide application of dual-energy CT and continuous technological innovation, different post-processing and analysis algorithms have been continuously developed. Virtual monoenergetic reconstruction is one of the important post-processing applications of dual-energy CT. It is generated by virtually calculating the CT value of the substance under each single energy through high and low dual-energy CT scanning [3]. The third generation of dual-source dual-energy CT (German Siemens SOMATOM Force CT) can calculate single-energy images of any energy level ranging from $40 \mathrm{keV}$ to $190 \mathrm{keV}$. Because substances with high atomic number such as iodine contrast agent have strong ability to absorb low energy $\mathrm{x}$-ray photons, in low energy single-energy spectrum images, tissues such as blood vessels and lesions enhanced by contrast agent have better contrast than those under ordinary single-energy CT scanning, which can be used to optimize the display of lesions. However, since the lower the energy is, the lower the penetration ability of $\mathrm{x}$-ray photons is and the higher the noise is. In addition, the signal-to-noise ratio of the images will also be lower, and even affect the diagnosis. Therefore, the traditional virtual smonoenergetic reconstructions technology (Mono_E) has not overcome the noise of low energy single-energy image, which affects its wider application.

Mono+, the new generation of virtual smonoenergetic reconstructions technology of dual-source dual-energy CT, overcomes the shortcomings of tradi- 
tional virtual monoenergetic reconstructions technology Mono_E and highlights its advantages in noise reduction. In recent years, the clinical application of this new technique in abdomen has also been continuously reported [4] [5] [6]. It is believed to bea promising technique that can improve image contrast, reduce noise, and improve image quality and the detection rate of small lesions. At present, the application research of dual-source CT in the diagnosis of esophageal cancer is mainly reflected in the application of reducing the radiation dose of patients [7], the characteristics of energy spectrum curve, iodine base map and other aspects in the fields such as TNM staging of esophageal cancer, differential diagnosis of metastatic lymph nodes and other fields [8] [9]. However, the research on virtual single energy in improving the quality of CT imaging of esophageal cancer patients is relatively rare. The purpose of this study was to explore the role of virtual single Energy reprocessing of dual-source dual-Energy CT in improving CT imaging quality of esophageal carcinoma. Through dual-source dual-energy CT examination on a group of pancreatic cancer patients, Davide Bellini and other scholars found that when the energy value was less than $70 \mathrm{keV}$, Mono+ could overcome the significant noise conventional of Mono images to improve the quality of the image. The objective image quality evaluation showed that the optimal contrast noise ratio could be obtained in the Mono+ $40 \mathrm{keV}$ energy level, and the subjective image quality evaluation considered that the ideal image quality could be obtained in Mono+ $55 \mathrm{keV}$ energy level [10]. Similarly, studies of Claudia Frellese and other scholars on the image quality of the parts between pancreatic cancer lesions and normal pancreatic tissue also found that the optimal image quality was achieved in Mono+ $55 \mathrm{keV}$ energy level [6]. Therefore, this study chose $55 \mathrm{keV}$ to compare Mono_E images and Mono+ images with conventional $100 \mathrm{kV}$ images. The results showed that the noise of Mono_E $55 \mathrm{keV}$ images was significantly higher than that of $100 \mathrm{kV}$ images, and the signal-to-noise ratio was significantly decreased. The subjective image quality was also significantly reduced. The study showed that the traditional virtual monoenergetic reconstructions technique Mono_E could improve the contrast between normal esophageal tissue and tumor lesions, but the image quality was reduced because the noise was too high; Mono+, the new generation of virtual monoenergetic reconstructions technology, significantly improved the contrast between normal esophagus and tumor lesions and the noise was not elevated. As a result, the signal-to-noise ratio of the image and the contrast noise of the tumor was improved (Figure 1).

To sum up, Mono+ technology, a new generation of virtual monoenergetic reconstructions technology of dual-source dual-energy CT, could significantly reduce image noise and improve imaging quality in CT imaging of esophageal cancer. Selection of an appropriate low $\mathrm{keV}$ virtual monoenergetic reconstructions image could obviously improve the contrast between tumor and normal esophageal tissue. This could improve the detection rate of esophageal cancer, and more clearly define the scope of esophageal tumor invasion, which has 


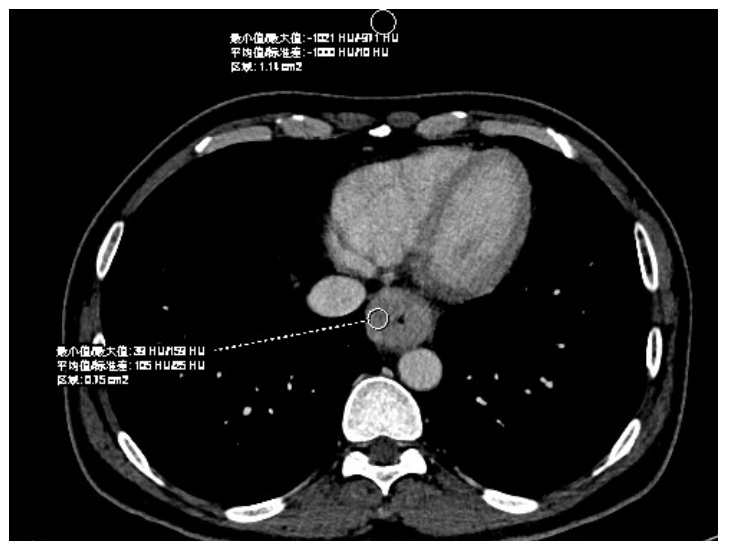

(a)

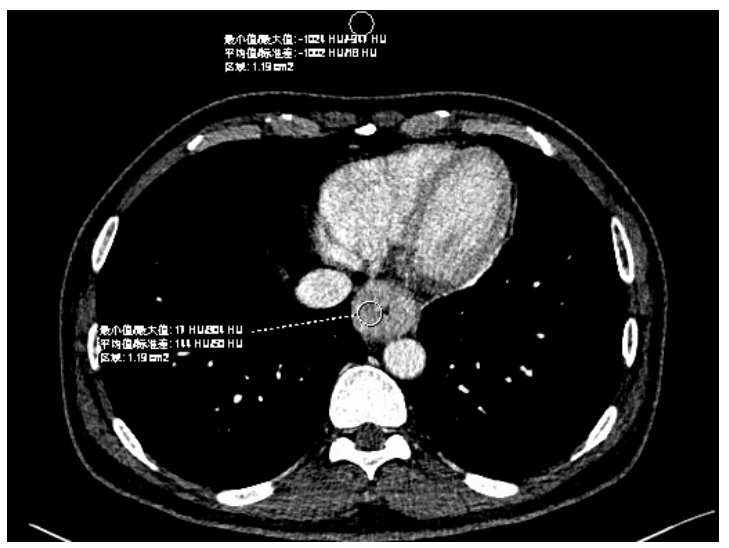

(b)

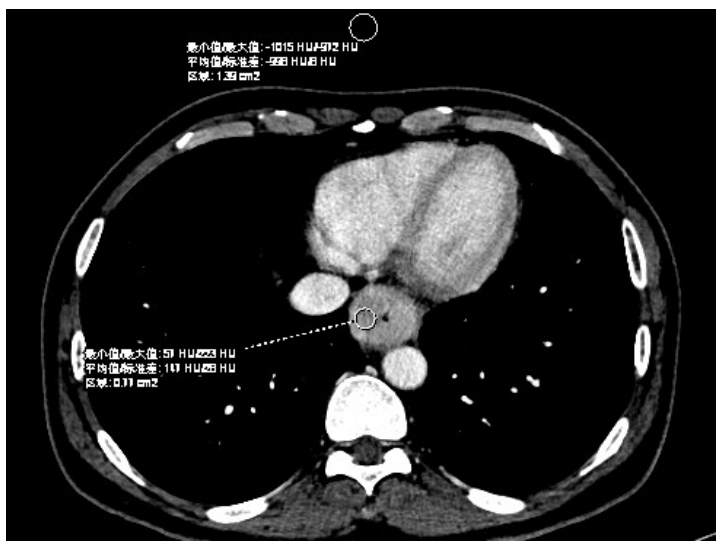

(c)

Figure 1. (a) to (c) were images of $100 \mathrm{Kv}$, Mono_E $55 \mathrm{keV}$ and Mono+e $55 \mathrm{keV}$ respectively. It was shown that Mono_E $55 \mathrm{keV}$ images were significantly higher than that of $100 \mathrm{kV}$ images, but the image noise and the air noise were also significantly elevated, which led to the decrease of imaging quality; the CT value of the esophageal tumor location in E55 keV images was significantly higher than that of $100 \mathrm{kV}$ images, but the noise was not found to be different. The air noise was decreased and the subjective imaging quality was improved.

important clinical significance for preoperative evaluation and treatment of esophageal cancer. 


\section{Conflicts of Interest}

The authors declare no conflicts of interest regarding the publication of this paper.

\section{References}

[1] Ferlay, J., Shin, H.R., Bray, F., et al. (2010) Estimates of Worldwide Burden of Cancer in 2008: GLOBOCAN 2008. International Journal of Cancer, 127, 2893-2917. https://doi.org/10.1002/ijc.25516

[2] Merkow, R.P., Bilimoria, K.Y., Keswani, R.N., et al. (2014) Treatment Trends, Risk of Lymph Node Metastasis, and Outcomes for Localized Esophageal Cancer. Journal of the National Cancer Institute, 106, 133. https://doi.org/10.1093/jnci/dju133

[3] Lu, G.M. and Zhang, L.J. (2016) Guide to Clinical Application of Dual-Energy CT. People's Health Publishing House, No. 1, 6-8.

[4] Schabel, C., Bongers, M., Sedlmair, M., et al. (2014) Assessment of the Hepatic Veins in Poor Contrast Conditions Using Dual Energy CT: Evaluation of a Novel Monoenergetic Extrapolation Software Algorithm. Fortschr Röntgenstr, 186, 591-597.

[5] Andrew, D., Hardie, M.D., Melissa, M., et al. (2015) Application of an Advanced Image-Based Virtual Monoenergetic Reconstruction of Dual Source Dual-Energy CT Data at Low keV Increases Imaging quality for Routine Pancreas Imaging. Journal of Computer Assisted Tomography, 39, 716-720. https://doi.org/10.1097/RCT.0000000000000276

[6] Frellesena, C., Fesslera, F., Andrew, D., et al. (2015) Dual-Energy CT of the Pancreas: Improved Carcinoma-to-Pancreascontrast with a Noise-Optimized Monoenergetic Reconstruction Algorithm. European Journal of Radiology, 84, 2052-2058. https://doi.org/10.1016/j.ejrad.2015.07.020

[7] Li, Q.W., Yu, T., Yu, M., et al. (2017) Prospective Study on the Effect of in Dual-Source Dual-Energy Single-Phase Enhanced CT Scan on Radiation Dose of Esophageal Cancer Patients. Chinese Journal of Digestive Surgery, 16, 527-532.

[8] Xu, X.Q. (2018) Diagnostic Value and Application of CT for Esophageal Cancer. Imaging Research and Medical Application, 2, 105-106.

[9] Liu, X.J., Miao, C.C., Zhou, S., et al. (2016) Application Value of Dual-Source CT Energy Spectrum Curve in Diagnosis and Pathological Classification of Esophageal Cancer. Journal of Clinical and Experimental Medicine, 15, 692-695.

[10] Bellini, D., Gupta, S., Ramirez-Giraldo, J.C., Marin, D., et al. (2017) Use of a Noise Optimized Monoenergetic Algorithm for Patient-Size Independent Selection of an Optimal Energy Level during Dual-Energy CT of the Pancreas. Journal of Computer Assisted Tomography, 41, 39-47. 